\title{
Mortalidade Compensada por Doenças Cardiovasculares no Período de 1980 a 1999 - Brasil
}

\author{
Balanced Cardiovascular Disease Mortality from 1980 to 1999 - Brazil
}

Gláucia Maria Moraes Oliveira, Nelson Albuquerque Souza e Silva, Carlos Henrique Klein Universidade Federal do Rio de Janeiro, Escola Nacional de Saúde Pública e Secretaria de Estado de Saúde do Rio de Janeiro - Rio de Janeiro, RJ

\section{Objetivo}

Avaliar comparativamente a evolução das mortalidades por doenças do aparelho circulatório (DAC), doenças isquêmicas do coração (DIC) e cerebrovasculares (DCBV), no estados do Rio de Janeiro (RJ), São Paulo (SP) e Rio Grande do Sul (RS), e suas capitais, no período 1980-1999.

\section{Métodos}

Os dados relativos a óbitos por DAC provieram do Datasus, e os das populações, do IBGE. Calcularam-se taxas de mortalidade brutas e ajustadas, por sexo e idade, pelo método direto (população padrão: maiores de vinte anos do Estado do RJ, em 2000). Em razão do crescimento relevante da mortalidade por causas mal definidas no município e no Estado do RJ, a partir de 1990, procedeu-se à compensação dos óbitos preliminares aos ajustamentos. As tendências foram analisadas com regressões lineares.

\section{Resultados}

Os declínios anuais da mortalidade, compensados e ajustados, variaram de $-11,3$ óbitos por DAC, por cem mil habitantes, no município e no Estado do RJ, até -7,4 no município de SP. As DIC foram semelhantes no Estado e município do RJ e em Porto Alegre, sendo menores no município de SP (-2,5 óbitos por cem mil habitantes). Nas DCBV, a variação observada foi de $-6,0$ a $-2,8$ óbitos por cem mil habitantes, no Estado do RJ e em Porto Alegre, respectivamente.

\section{Conclusão}

Observaram-se declínios das taxas de mortalidade compensadas e ajustadas por DAC, DIC e DCBV, no período 1980-1999, nos três estados e capitais. No RJ, Estado e município, os declínios das DIC foram nítidos a partir de 1990, enquanto as DCBV mostraram quedas em todo o período.

\section{Palavras-chave}

tendência, mortalidade, doenças cardiovasculares

\section{ОвJECTIVE}

To compare trends in mortality rates from cardiovascular diseases (CVD), ischemic heart diseases (IHD) and cerebrovascular diseases (CBVD) in the States of Rio de Janeiro (RJ), São Paulo (SP) and Rio Grande do Sul (RS) and respective capitals from 1980 to 1999.

\section{Methods}

Data regarding CVD deaths were obtained from Datasus, and those regarding populations were obtained from IBGE. Crude and sex and age-adjusted mortality rates were calculated using the direct method (standard population: State of Rio de Janeiro's population twenty years of age or older in 2000). Because of the relevant increase in mortality from ill-defined causes in the city and State of RJ as of 1990, the deaths were balanced prior to adjustments. The trends were analyzed using linear regressions.

\section{RESULTS}

Annual declines of balanced and adjusted mortality ranged from -11.3 CVD deaths/100,000 inhabitants in the city and State of RJ to -7.4 in the city of SP. IHD mortality rates were similar in the State and city of $R J$ and in Porto Alegre, and lower in the city of SP (-2.5 deaths/100,000 inhabitants). CBVD mortality rates ranged from -6.0 to 2.8 deaths $/ 100,000$ inhabitants in the State of RJ and in Porto Alegre, respectively.

\section{Conclusion}

A decline in balanced and adjusted CVD, IHD and CBVD mortality rates was observed from 1980 to 1999 in the three States and capitals. In the State and city of RJ declines in IHD were clear as of 1990, whereas declines in CBVD occurred throughout the period studied.

\section{KEYWORDS}

trend, mortality, cardiovascular diseases

Correspondência: Gláucia Maria Moraes Oliveira - Rua Fadel Fadel, 112/1204 - 22430-170 - Rio de Janeiro, RJ 
A partir do final da década de 1960 e início dos anos 1970, um declínio da mortalidade por doenças do aparelho circulatório (DAC) começou a ser observado nos países industrializados, principalmente nos Estados Unidos, no Canadá e nos países do Oeste Europeu. Apesar da tendência de queda dessas taxas de mortalidade, as DAC são as principais causas de morte nos países desenvolvidos ou em desenvolvimento ${ }^{1,2}$, e também no Brasil $^{3,4}$. Assim, no ano de 1998, as DAC foram responsáveis por $26,5 \%$ dos óbitos no Brasil ${ }^{5}$.

No Brasil, essa tendência de queda das taxas de mortalidade por DAC foi constatada em estudos realizados no Estado e município de São Paulo6,7 e, posteriormente, em capitais como Salvador ${ }^{8}$ e Goiânia ${ }^{9}$. Melhor controle de fatores de risco, especialmente hipertensão arterial sistêmica, e novas técnicas diagnósticas, além de avanços no campo terapêutico foram considerados os principais responsáveis pela redução dessas taxas de mortalidade ${ }^{10-14}$.

Na região Sudeste, em 1996, as DAC representaram $30,8 \%$ das mortes, seguidas por causas externas com $13,5 \%$ e neoplasias com $12,4 \%^{5}$. Dentre as DAC, destacam-se as doenças cerebrovasculares (DCBV) e as doenças isquêmicas do coração (DIC), que em 1998 compuseram mais de $60 \%$ das taxas de óbitos por DAC, no Brasil ${ }^{4}$. No Estado do Rio Grande do Sul, bem como em sua capital, Porto Alegre, as DAC também foram as principais causas de mortalidade em $30 \%$ a $35 \%$ dos óbitos nos últimos trinta anos ${ }^{15}$.

Souza e cols. ${ }^{16}$, estudando a tendência do risco de morte por DAC nas cinco regiões do Brasil, no período de 1979 a 1996, corroboraram a maioria dos achados descritos acima. Esses autores demonstraram uma tendência de queda de mortalidade por DAC nas regiões Sul, Sudeste e Norte, em todas as faixas etárias e em ambos os sexos.

Mansur e cols. ${ }^{3}$ estudaram, posteriormente, a mortalidade por DAC, DIC e DCBV em onze capitais do Brasil, no período de 1980 a 1998, das cinco grandes regiões. Observaram quedas na maioria das capitais. 0 Rio de Janeiro apresentou reduções dos riscos de morte por DIC e DCBV, bem como Porto Alegre. Entretanto, São Paulo apresentou um discreto aumento nas DIC, em ambos os sexos, na faixa etária de trinta a 39 anos entre os homens e, entre as mulheres, de quarenta a 59 anos. Os autores salientaram as relevantes diminuições nas DAC, DIC e DCBV no Rio de Janeiro.

Embora esses autores mencionem as altas taxas de mortalidade por causas mal definidas nas regiões Nordeste, Norte e Centro-Oeste ${ }^{16}$, não referem a crescente mortalidade por esse grupo de causas no Rio de Janeiro a partir de 1990 e sua influência nas tendências observadas nas taxas de mortalidade por causas definidas.

O desenvolvimento de um sistema de informação em saúde, composto de bases de dados confiáveis para análise, é fundamental para a tomada de decisões gerenciais buscando definir prioridades, estratégias e ações voltadas para a melhoria de saúde da população ${ }^{17}$.
Este estudo tem como objetivo principal delinear o comportamento evolutivo da mortalidade por DAC e suas frações, DIC e DCBV, no Estado do Rio de Janeiro (RJ), comparando-a com a sua evolução nos Estados do Rio Grande do Sul (RS) e de São Paulo (SP), utilizando uma casuística histórica dos anos de 1980 a 1999, por meio do emprego de metodologia uniforme de análise dos dados.

\section{MÉTodos}

0 estudo tem delineamento ecológico de série histórica, com dados dos anos de 1980 a 1999, o último ano com dados disponíveis na época do estudo. Analisouse a mortalidade por DAC nos estados do Rio de Janeiro, São Paulo e Rio Grande do Sul, bem como em suas respectivas capitais, na população adulta de vinte anos ou mais. As populações totais, de adultos e de idosos, dos locais estudados, no ano de 2000, podem ser observadas na tabela I.

Os dados referentes às populações utilizadas para os cálculos das taxas de mortalidade foram obtidos do Instituto Brasileiro de Geografia e Estatística (IBGE) com base nos censos de 1970, 1980, 1991 e 2000, e da contagem populacional de $1996^{18}$, utilizando o método de Lagrange para os cálculos das populações em $1^{\circ}$ de julho para os anos intercensitários de 1980 a $1999^{19}$.

Os dados relativos às freqüências anuais de mortes por DAC, no período entre 1980 e 1999, foram obtidos do Sistema Datasus, Ministério da Saúde ${ }^{20}$. Utilizaram-se os códigos da $9^{a}$ Conferência de Revisão da Classificação Internacional de Doenças de $1975^{21}$ (CID 9) para os óbitos de 1980 a 1995, com os seguintes códigos: DIC- 410 a 414, DCBV- 430 a 438 e DAC- 390 a 459 . Para os óbitos a partir de 1996 , até 1999 , foi utilizada a $10^{\text {a }}$ Conferência de Revisão da Classificação Internacional de Doenças $^{22}$ de 1995(CID 10) com os códigos de DIC- I20 a I25, DCBV- 160 a 69 e DAC- 110 a I82.9.

A população foi estratificada de acordo com o sexo e os grupos etários (20 a 29; 30 a 39; 40 a 49; 50 a 59; 60 a 69; 70 a 79 e 80 ou mais), sendo então calculadas as taxas de mortalidade brutas e ajustadas (por sexo e idade) pelo método direto. 0 ajustamento teve como população padrão a dos maiores de vinte anos no Estado do Rio de Janeiro no ano de 2000, com base no censo realizado nesse mesmo ano. Esse procedimento se justifica pelas diferentes distribuições de sexo e idade observadas nos locais estudados (tab.l).

Observaram-se a partir de 1990 aumentos relevantes da mortalidade por causas mal definidas no Estado e no município do Rio de Janeiro, o mesmo não ocorrendo nos estados de São Paulo e Rio Grande do Sul, e suas respectivas capitais. Por esse motivo, decidiu-se adicionar aos óbitos certificados pelas causas definidas de interesse (DIC, DCBV e DAC) uma determinada proporção de óbitos de causas mal definidas, em cada grupo, de acordo com o sexo e a idade. Essa proporção é aquela observada da 


\begin{tabular}{|c|c|c|c|}
\hline População & $\begin{array}{c}\text { Total } \\
n\end{array}$ & $\begin{array}{c}20 \text { anos ou mais } \\
n(\%)\end{array}$ & $\begin{array}{c}60 \text { anos ou mais } \\
n(\%)\end{array}$ \\
\hline Estado do Rio de Janeiro & 14.391 .282 & $9.455 .351(65,7 \%)$ & $1.540 .754(10,7 \%)$ \\
\hline Estado do Rio Grande do Sul & 10.187 .798 & $6.566 .633(64,5 \%)$ & $1.065 .484(10,5 \%)$ \\
\hline Estado de São Paulo & 37.032 .403 & $23.648 .451(63,9 \%)$ & $3.316 .957(9,0 \%)$ \\
\hline Município do Rio de Janeiro & 5.857 .904 & $4.028 .858(68,8 \%)$ & $751.637(12,8 \%)$ \\
\hline Município de Porto Alegre & 1.360 .590 & $921.796(67,7 \%)$ & $160.541(11,8 \%)$ \\
\hline Município de São Paulo & 10.434 .252 & $6.848 .778(65,6 \%)$ & $972.199(9,3 \%)$ \\
\hline
\end{tabular}

causa definida (DIC, DCBV ou DAC), em relação a todos os óbitos, excluindo-se os mal definidos. A suposição é a de que as distribuições das causas de óbitos entre os mal definidos são semelhantes às observadas entre os óbitos de causas definidas. Esse procedimento foi utilizado em todos os locais, não apenas no Rio de Janeiro. Portanto, podemos resumir esse procedimento em uma fórmula como a que se segue: $X=X+M * X /(T-M)$ em que $X$ é o número de óbitos pela causa específica (DAC, DIC ou DCBV), M é o número de óbitos por causas mal definidas, T é o número de óbitos por todas as causas, e $X_{c}$ é o número compensado de óbitos pela causa específica.

Depois dessa compensação é que se procedeu ao ajustamento por padronização; portanto, passamos a denominar as taxas de mortalidade resultantes de "compensadas e ajustadas".

Para a análise de tendências de mortalidade utilizaramse as taxas de mortalidade compensadas e ajustadas, estimando-se as taxas com modelos de regressão linear, considerando as taxas de mortalidade compensadas e ajustadas por DAC, DIC e DCBV observadas como variáveis dependentes $\mathbf{Y}$, e os anos calendários do estudo como variáveis independentes $\mathbf{X}$. As taxas médias do período estudado correspondem ao ponto médio entre os anos de 1989 e 1990.

Os modelos ficaram assim representados: $\hat{\mathbf{Y}}=\mathbf{a}+\mathbf{b X}$, onde $\hat{Y}$ é a taxa estimada compensada e ajustada; a é a taxa média do período; b é a variação anual média (coeficiente de inclinação da reta) eX é o ano (ano $0=$ 1980 e ano 19 = 1999).
As proporções das variâncias totais explicadas pelos modelos são expressas em $r^{2}$ (variação de 0 a 1 ). As porcentagens de variação anual foram calculadas a partir das razões b/a.

Para o ajustamento de taxas e demais manobras quantitativas foi utilizado o programa STATA ${ }^{23}$.

\section{Resultados}

O município do Rio de Janeiro, conforme mostrado na tabela I, apresenta perfil etário com porcentual de idosos maior do que as demais capitais, São Paulo e Porto Alegre, o mesmo ocorrendo na comparação entre os estados.

A tabela II apresenta as taxas brutas de mortalidade nos estados, e suas respectivas capitais, das DAC, DIC, $\mathrm{DCBV}$, mal definidas e de todas as causas. Observamos decréscimos das taxas brutas de DAC, DCBV e DIC nos três estados e suas capitais. O Estado e o município do Rio de Janeiro detinham as maiores taxas brutas na primeira década, apresentando-se no final da segunda década com todas essas taxas em níveis mais elevados que os dois outros estados e capitais, exceto para as DIC.

Os óbitos por causas mal definidas apresentaram distribuições desiguais nos estados e municípios estudados. No Estado e município de São Paulo as taxas de mortalidade permaneceram constantes. No Rio Grande do Sul e Porto Alegre as mortalidades brutas por causas mal definidas caíram em mais de $50 \%$ da década de 1980 1989 para a seguinte, porém no Estado do Rio de Janeiro essa mortalidade aumentou mais de duas vezes, e no

\begin{tabular}{|c|c|c|c|c|c|c|c|}
\hline \multirow[t]{2}{*}{ Causa } & \multirow[t]{2}{*}{ Década } & \multicolumn{3}{|c|}{ Estados } & \multicolumn{3}{|c|}{ Capitais } \\
\hline & & RJ & SP & RS & Rio & S. Paulo & P. Alegre \\
\hline \multirow[t]{2}{*}{ DIC } & 1980-1989 & 165,2 & 126,5 & 133,9 & 192,2 & 135,2 & 169,0 \\
\hline & 1990-1999 & 128,6 & 113,8 & 130,3 & 150,8 & 134,0 & 151,0 \\
\hline \multirow[t]{2}{*}{ DCBV } & 1980-1989 & 188,6 & 128,9 & 138,5 & 190,2 & 114,9 & 124,1 \\
\hline & 1990-1999 & 150,7 & 104,3 & 127,4 & 149,5 & 98,8 & 118,7 \\
\hline \multirow[t]{2}{*}{ DAC } & 1980-1989 & 493,4 & 377,7 & 386,6 & 522,0 & 361,2 & 405,3 \\
\hline & 1990-1999 & 412,3 & 337,6 & 366,1 & 434,7 & 347,5 & 380,6 \\
\hline Causas & 1980-1989 & 45,3 & 50,9 & 83,4 & 26,0 & 11,0 & 18,5 \\
\hline Mal Definidas & 1990-1999 & 110,5 & 50,2 & 44,2 & 110,6 & 10,5 & 11,4 \\
\hline Todas as & 1980-1989 & $1.098,8$ & 907,5 & 937,9 & $1.138,9$ & 861,4 & 961,3 \\
\hline Causas & 1990-1999 & $1.157,2$ & 956,2 & 988,7 & $1.237,2$ & 957,2 & $1.053,0$ \\
\hline
\end{tabular}


município do Rio de Janeiro quadruplicou da primeira para a segunda décadas. Ainda mais, os níveis das taxas de mortalidade por causas mal definidas variaram consideravelmente entre os estados e municípios, apresentando o Estado e o município do Rio de Janeiro as mais elevadas, especialmente na década de 1990-1999, em que as taxas do município do Rio de Janeiro são dez vezes maiores do que nas outras duas capitais (figs. 1 e 2).

Podemos observar que, enquanto o município e o Estado de São Paulo mantiveram taxas estáveis de mortalidade por causas mal definidas, ocorreu queda progressiva no
Estado do Rio Grande do Sul e Porto Alegre, e elevação no município e Estado do Rio de Janeiro a partir de 1990. Em Porto Alegre, a estabilidade dessas taxas só foi alterada para mais nos últimos anos da década de 1980, voltando a cair no início da década de 1990-1999.

A tabela III apresenta os valores estimados pelos modelos de regressão linear das taxas de mortalidade compensadas e ajustadas. Os modelos lineares foram capazes de explicar a variância total de forma satisfatória $\left(r^{2}=0,77\right.$ a 0,95$)$.

Observamos que os declínios anuais estimados das taxas de mortalidade, compensadas e ajustadas, tanto nas capi-
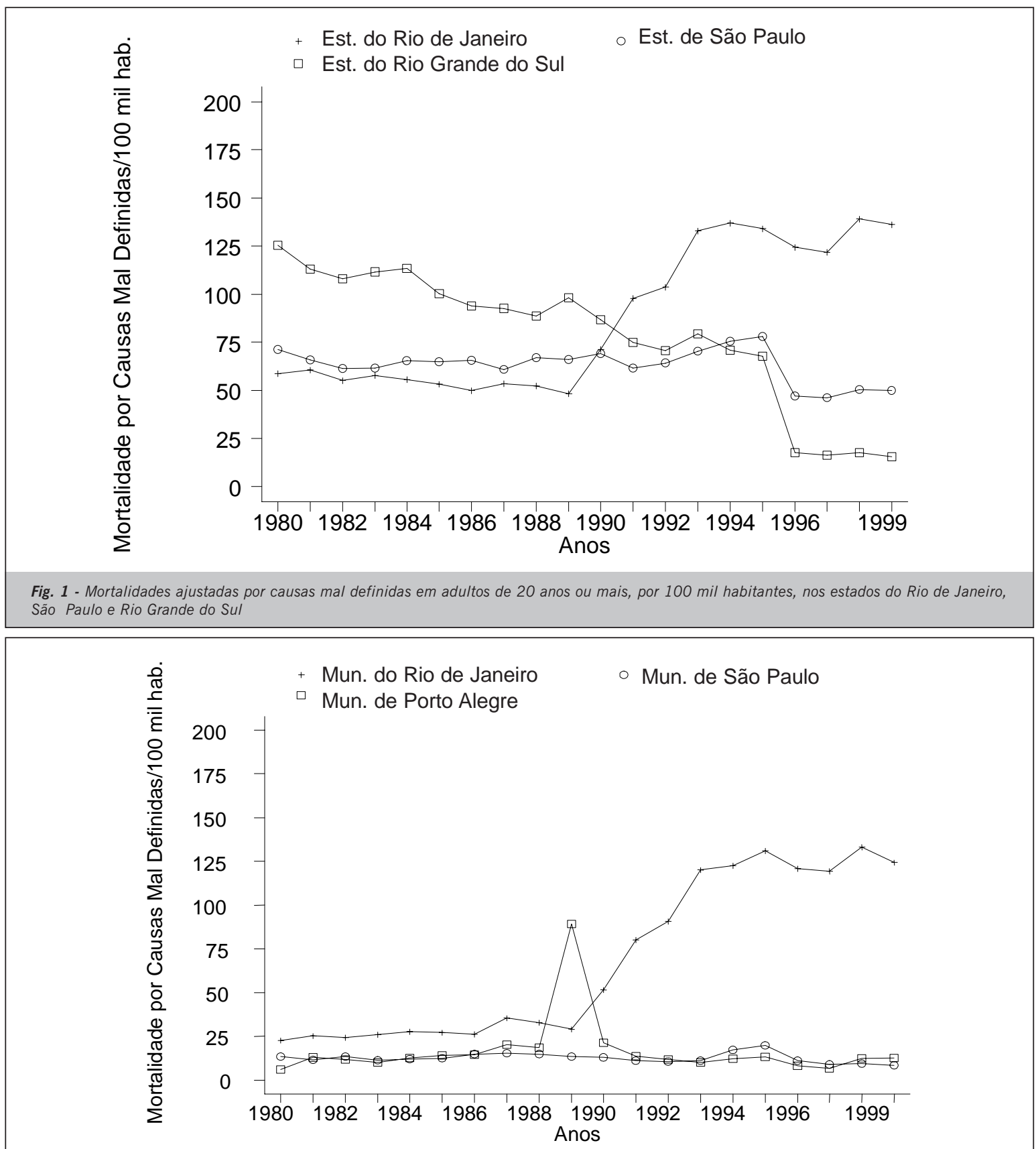
tais quanto nos estados do Rio de Janeiro, São Paulo e Rio Grande do Sul, variaram de $-11,3$ óbitos por DAC, por cem mil habitantes, no município e no Estado do Rio de Janeiro, a -7,4 óbitos, por cem mil habitantes, no município de São Paulo. Nas DIC os declínios foram semelhantes no Estado do Rio de Janeiro e sua capital, bem como em Porto Alegre, sendo o menor no município de São Paulo (2,5 óbitos por cem mil habitantes). Nas DCBV, a variação observada é de $-6,0$ óbitos, por cem mil habitantes, no Estado do Rio de Janeiro, até -2,8 óbitos, por cem mil habitantes, em Porto Alegre (tab. III).

Os porcentuais de variação anuais das taxas de mortalidade estimadas apresentaram diminuição das DAC, mesmo de suas parcelas DIC e DCBV. As DCBV predominaram no Estado (217,4/100 mil habitantes) e município do Rio de Janeiro (192,9/100 mil habitantes) em relação aos demais estados e capitais, o mesmo acontecendo com as DIC em menor grau.
As figuras 3 a 6 apresentam as taxas de mortalidade compensadas e ajustadas nos estados e capitais, por DIC e DCBV.

O Estado e o município do Rio de Janeiro apresentaram as maiores taxas de mortalidade por DAC no período, seguidos pelo Estado e o município de São Paulo, ficando o Estado do Rio Grande do Sul e sua capital Porto Alegre com as taxas mais baixas. O Estado do Rio de Janeiro apresentou as maiores taxas de mortalidade, compensadas e ajustadas, por DIC até 1990, aproximando-se dos outros estados no final da série histórica (fig. 3). As DIC apresentaram taxas de mortalidade, compensadas e ajustadas, semelhantes nas capitais.

O Estado do Rio de Janeiro apresentou as maiores taxas de mortalidade, compensadas e ajustadas, por DCBV, ao longo de todo o período estudado. Porém, a tendência de declínio dessas taxas foi um pouco mais acentuada do que nos estados de São Paulo e do Rio

Tabela III - Estatísticas de mortalidade anuais compensadas e ajustadas, estimadas por DAC, DIC e DCBV nos estados do Rio de Janeiro, Rio Grande do Sul e São Paulo e suas respectivas capitais, por cem mil habitantes nos maiores de vinte anos de idade, no período de 1980 a 1999

\begin{tabular}{|c|c|c|c|c|c|c|c|}
\hline \multirow[t]{2}{*}{ Causas } & \multirow[t]{2}{*}{ Estatísticas $\left({ }^{*}\right)$} & \multicolumn{3}{|c|}{ Estados } & \multicolumn{3}{|c|}{ Capitais } \\
\hline & & RJ & SP & RS & Rio & S. Paulo & P. Alegre \\
\hline \multirow[t]{4}{*}{ DIC } & Média(M) & 183,4 & 168,8 & 166,2 & 192,2 & 185,2 & 188,2 \\
\hline & Dif. Anual (DA) & $-4,3$ & $-3,3$ & $-2,6$ & $-4,6$ & $-2,5$ & $-4,5$ \\
\hline & $D A / M$ & $-2,3 \%$ & $-2,0 \%$ & $-1,6 \%$ & $-2,4 \%$ & $-1,3 \%$ & $-2,4 \%$ \\
\hline & $r^{2}$ & 0,85 & 0,95 & 0,81 & 0,82 & 0,83 & 0,77 \\
\hline \multirow[t]{4}{*}{ DCBV } & Média(M) & 217,4 & 167,3 & 173,6 & 192,9 & 148,2 & 142,7 \\
\hline & Dif. Anual (DA) & $-6,0$ & $-5,1$ & $-4,3$ & $-5,9$ & $-4,0$ & $-2,8$ \\
\hline & $\mathrm{DA} / \mathrm{M}$ & $-2,8 \%$ & $-3,0 \%$ & $-2,4 \%$ & $-3,0 \%$ & $-2,7 \%$ & $-2,0 \%$ \\
\hline & $r^{2}$ & 0,94 & 0,94 & 0,91 & 0,95 & 0,90 & 0,81 \\
\hline \multirow[t]{4}{*}{ DAC } & Média(M) & 572,3 & 506,5 & 484,1 & 538,2 & 486,4 & 460,4 \\
\hline & Dif. Anual (DA) & $-11,3$ & $-10,1$ & $-9,8$ & $-11,3$ & $-7,4$ & $-9,5$ \\
\hline & $\mathrm{DA} / \mathrm{M}$ & $-2,0 \%$ & $-2,0 \%$ & $-2,0 \%$ & $-2,1$ & $-1,5 \%$ & $-2,1 \%$ \\
\hline & $r^{2}$ & 0,91 & 0,92 & 0,88 & 0,92 & 0,87 & 0,88 \\
\hline
\end{tabular}

(*) Valores obtidos por regressão linear dos valores compensados e ajustados sobre os anos, sendo $M$ correspondente ao meio do período e $D A$ a inclinação da reta. M e DA em escala de óbitos por cem mil habitantes (compensação por acréscimo da proporção correspondente de óbitos mal definidos e ajustamento por sexo e grupo etário, pelo método direto, tendo como população padrão a do estado do Rio de Janeiro em 2000)

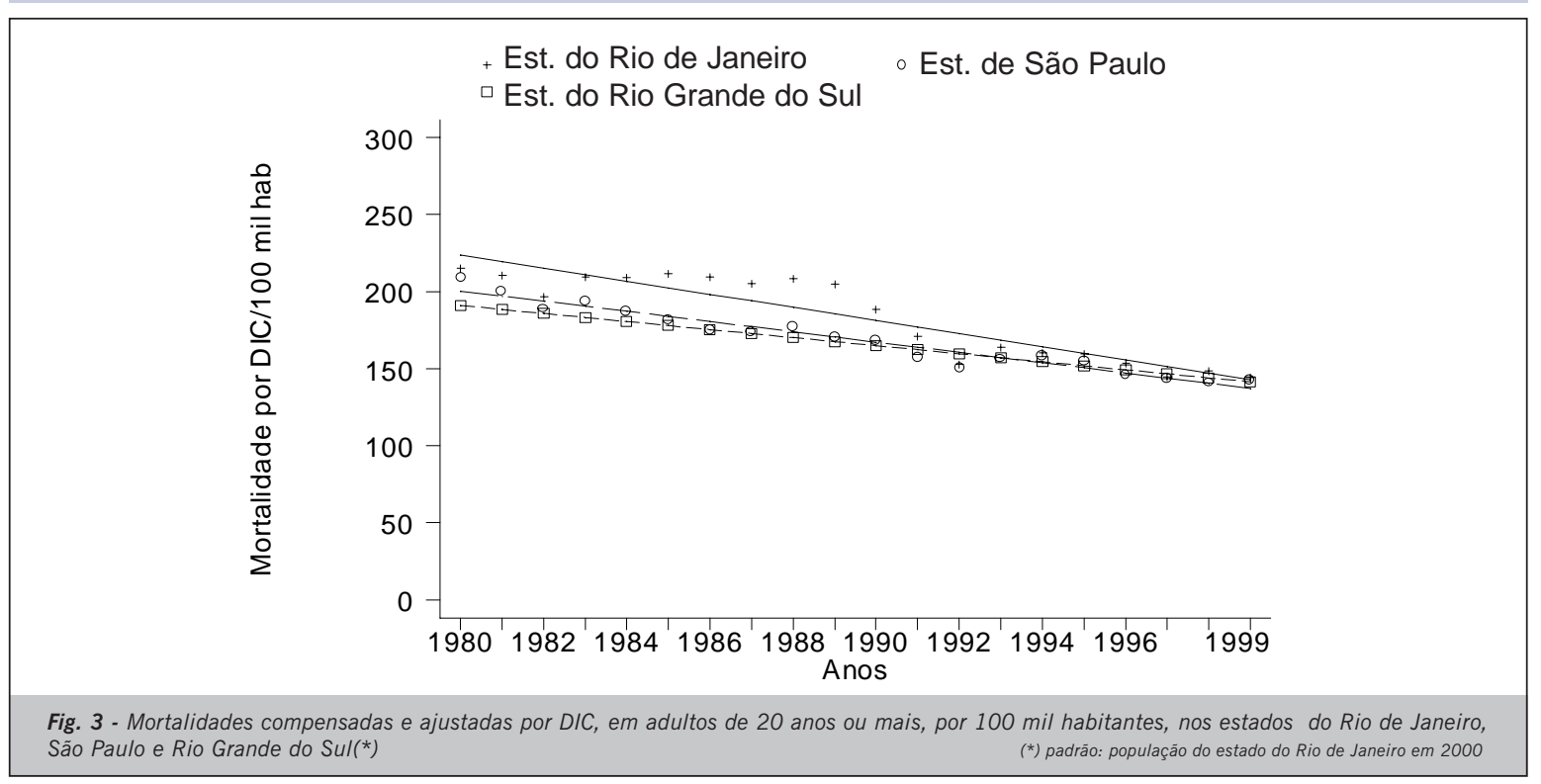



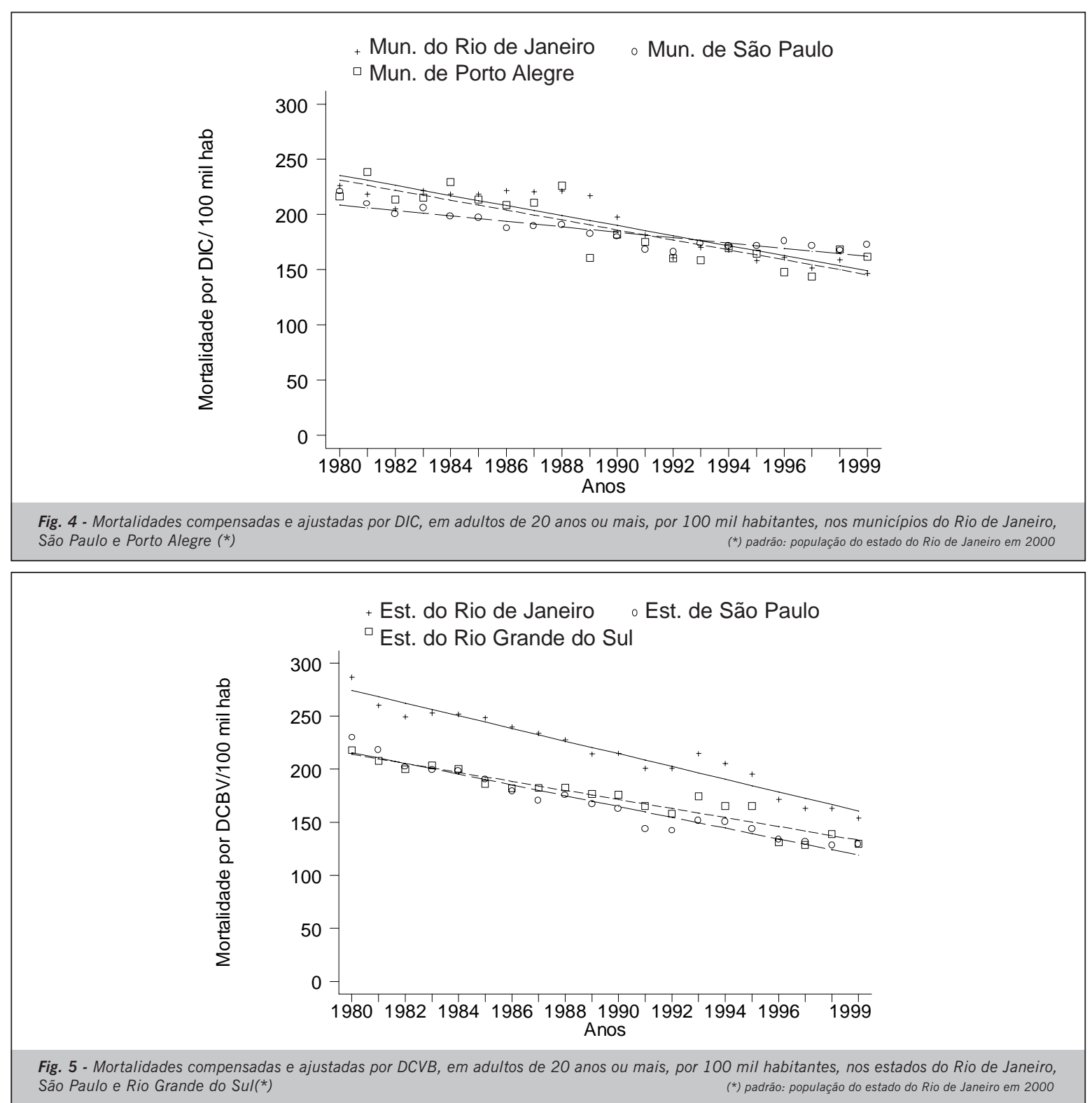

Grande do Sul (tab. III e fig. 5). Esses dois estados, São Paulo e Rio Grande do Sul, apresentaram taxas de mortalidade por DCBV bastante próximas. 0 mesmo padrão observado nos estados se repete na comparação das capitais (fig. 6). Apesar das taxas do município do Rio de Janeiro se apresentarem mais elevadas, sua tendência de declínio foi maior e no final do período as taxas de mortalidade por DCBV, compensadas e ajustadas, se aproximam.

\section{Discussão}

Nos últimos vinte anos, vários estudos mostraram tendências de declínio da mortalidade por DAC, inicialmente nos Estados Unidos, Canadá e Austrália no inicio da década de 1960, seguidos por Inglaterra,
1970, e França, Itália e Suécia na segunda metade dessa última década2,24,25.

No Brasil foram publicados diversos estudos sobre a mortalidade por DAC ${ }^{3,5,6-9,13,16,26}$ referentes a dados a partir da década de 1970. Laurenti e cols. ${ }^{27}$, em 1981 , sinalizaram para o aumento das DIC, que representavam as principais causas básicas de morte $(16,7 \%)$ em adultos de quinze a 74 anos no município de São Paulo. Concluíram também que as informações obtidas com os documentos de óbitos (DO) correlacionavam-se com razoável segurança com os dados obtidos na análise dos casos (sensibilidade de 75,3\% e especificidade de $95,8 \%$ ). Em outro estudo, esse mesmos autores chamaram a atenção para a preponderância das DCBV sobre as DIC em várias capitais brasileiras, aproximandose das taxas de óbito observadas por aquela causa no Japão, mesmo no sexo masculino ${ }^{28}$. 


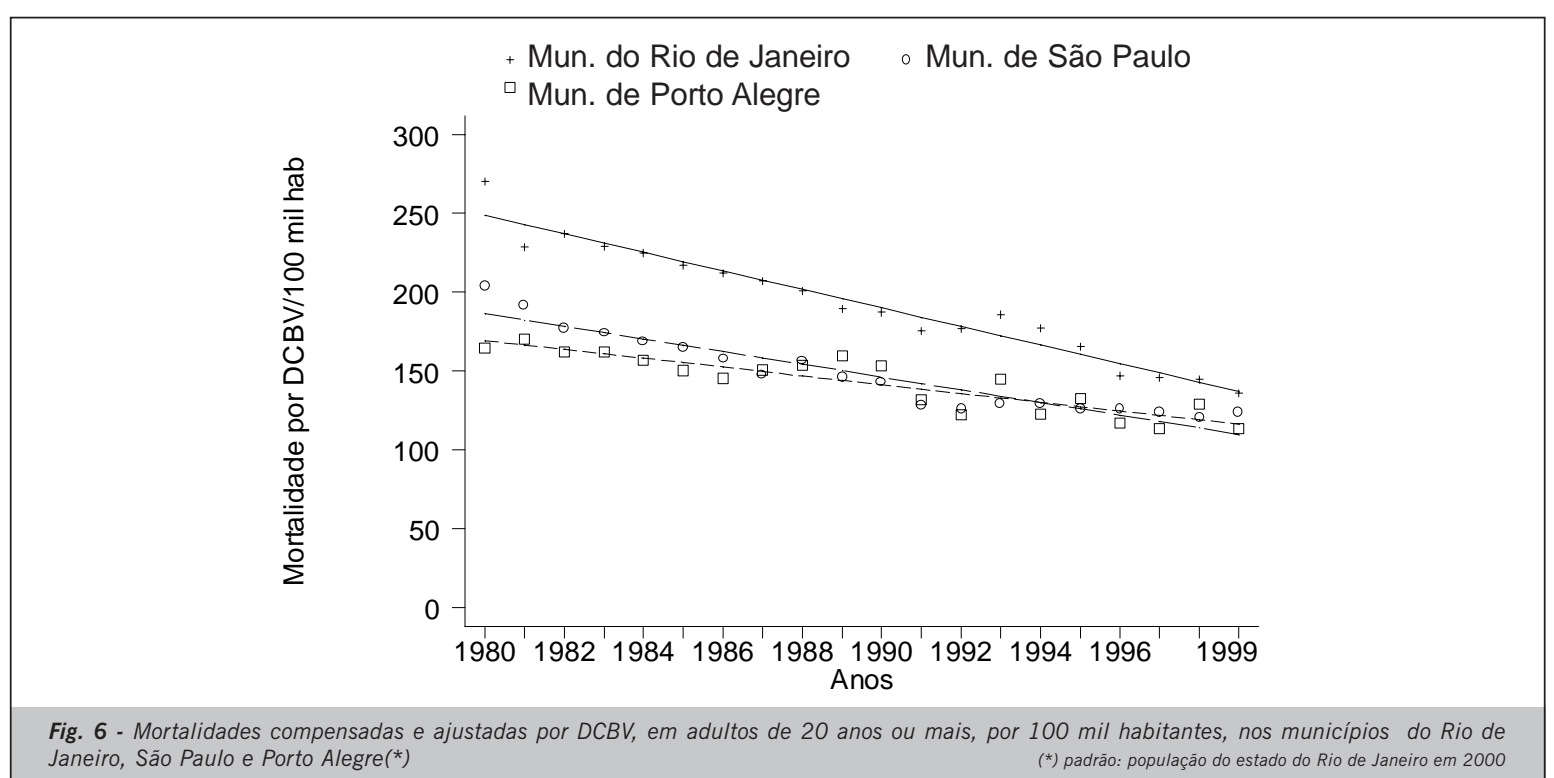

Chor e cols. ${ }^{26}$ compararam a mortalidade no Brasil com a verificada nos Estados Unidos, na Inglaterra e em Cuba em 1988, e constataram que cerca de $50 \%$ dos óbitos masculinos por DIC ocorriam antes dos 65 anos de idade em nosso país, contra $25 \%$ naqueles países. Entretanto, é preciso considerar que a população brasileira é mais jovem do que a desses países, como assinalaram os autores. A taxa de mortalidade por infarto agudo do miocárdio (IAM) no Brasil foi comparável à encontrada nos países do leste Europeu até a década de 1990, cujas taxas de mortalidade estavam entre as mais altas do mundo. 0 mesmo ocorreu em relação ao risco de morte por DCBV, que foi ainda maior no Brasil, comparado aos Estados Unidos, assemelhando-se também às taxas do Leste Europeu²6.

As DIC, em adultos maiores de vinte anos, de acordo com Lolio e cols. ${ }^{29}$, no município de São Paulo, apresentaram tendência de aumento nas taxas de mortalidade de 1950 a 1976, seguidas de declínio a partir daí, sendo maior no sexo feminino e nos mais jovens ${ }^{29}$. Nos anos de 1970 a 1983, a redução observada foi de $28 \%$ em média nas DIC e $16 \%$ nas DCBV, nas faixas etárias de quarenta a 69 anos. No entanto, essas taxas ainda eram elevadas quando comparadas com as de 27 países industrializados, após padronização com ajuste de idade em ambos os sexos ${ }^{13}$.

Durante o período de 1979 a 1989, as DIC e as DAC foram estudadas nas capitais de regiões metropolitanas do Brasil, observando-se declínios desiguais nas diversas capitais $^{30}$. No entanto, os autores apontaram aumentos nas taxas de mortalidade por DAC no Rio de Janeiro, $+1,4 \%$ nos homens e $+0,8 \%$ nas mulheres. Esse aumento de mortalidade também ocorreu por DIC no Rio de Janeiro, $+2,3 \%$ nos homens e $+2,5 \%$ nas mulheres. Note-se que o Rio de Janeiro foi a única capital estudada que apresentou tendência de aumento nas DAC e DIC nesse período.

Em nosso estudo, o município e o Estado do Rio de Janeiro apresentaram as maiores taxas de mortalidade por DAC e DCBV no período. Porém, a tendência de declínio dessas taxas, tanto no Estado quanto no município, foi mais acentuada no Rio do que nos demais estados e capitais, e no final do período as taxas se equivaliam nas capitais. Também, o Estado do Rio de Janeiro tinha as maiores taxas de mortalidade, compensadas e ajustadas, por DIC até 1990, aproximando-se dos outros estados no final da casuística histórica. Entretanto, as DIC apresentaram taxas de mortalidade, compensadas e ajustadas, semelhantes nas capitais. A observação atenta das mortalidades compensadas e ajustadas por DAC (tab. II) e DIC (tab. II, figs.3 e 4) mostra que tanto no Estado como no município do Rio de Janeiro essas taxas se mantiveram estáveis de 1980 a 1989, tendo iniciado seu declínio na década seguinte. Já as taxas de mortalidade compensadas e ajustadas por DCBV apresentaram declínio no Rio de Janeiro, Estado e município, a partir dos primeiros anos da casuística histórica dos dados disponíveis para nosso estudo (figs. 5 e 6). Portanto, é possível supor que o aumento das taxas de DAC e DIC se verificou durante a década de 1970, tendo ocorrido estabilização na década seguinte, e queda na década de 1990.

Lolio \& Laurenti ${ }^{31}$, estudando a mortalidade por DCBV em adultos maiores de vinte anos de idade, no município de São Paulo, de 1950 a 1981, observaram aumento na mortalidade nas décadas de 1950 e 1960 nos grupos acima de vinte anos, seguido de declínio entre 1970 e 1981, mais acentuado nos grupos etários acima dos sessenta anos em ambos os sexos. Esse declínio foi confirmado no presente trabalho no município e o no Estado de São Paulo com porcentuais de variação anual média de $-2,7 \%$ e -3,0\%, respectivamente.

Mansur e cols. ${ }^{3}$ observaram, no período de 1979 a 1996, tendências de queda nas mortalidades por DAC, DIC e DCBV em ambos os sexos no Brasil. As reduções observadas, por cem mil habitantes, foram: DIC - quase 
duas vezes maior nos homens, 2,94 contra 1,67 mortes/ ano nas mulheres; DCBV - cerca de três mortes/ano; DAC - oito mortes/ano, estas em ambos os sexos. 0 declínio das taxas de mortalidade por DIC ocorreu somente a partir de 1985, com queda progressiva até 1995, diferente dos Estados Unidos que a iniciaram em 1960 e mantiveram a queda desde então. Entretanto, segundo esses autores, as mortalidades por DCBV em homens e mulheres no Brasil foram sempre menores que as registradas nos países do estudo $\mathrm{MONICA}^{8}$, não se podendo dizer o mesmo para o Estado do Rio de Janeiro de acordo com nosso estudo.

Chamamos a atenção para a qualidade das informações sobre mortalidade, as quais podem ser avaliadas pela mortalidade por causas mal definidas. Destacamos o aumento desta no Estado e município do Rio de Janeiro a partir de 1990, o que aliás nos levou a trabalhar com as taxas de mortalidade compensadas, além de ajustadas por sexo e idade, pelas causas definidas de interesse (DAC, DIC e DCBV) para fazer comparações entre estados e municípios. Esse aumento no Estado e município do Rio de Janeiro pode estar relacionado com a portaria de 29 de janeiro de 1990, que determinou que "esgotadas todas as tentativas de se determinar a causa básica da morte e não havendo suspeita de óbito por causa violenta (acidente, homicídio ou suicídio), deverá ser declarada na parte I do atestado médico causa indeterminada" ${ }^{32}$. Essa portaria, provavelmente, é um dos fatores que influenciaram o preenchimento de documentos de óbito sem diagnóstico definido no Estado do Rio de Janeiro.

É possível, também, que a manobra que realizamos para compensar as "perdas" de óbitos por DAC, e suas parcelas, DIC e DCBV, ainda permita subestimar a verdadeira "força" da mortalidade por esses grupos de causas, especialmente no Rio de Janeiro. Neste estudo consideramos que as proporções de óbitos por DAC entre aqueles declarados por causa indeterminada, e portanto mal definidos, eram as mesmas que foram observadas, por DAC, entre todos os de causas definidas, por grupos de sexo e idade. Entretanto, observamos também que as DAC foram o único grande grupo de causas que sofreu uma queda relevante na mortalidade bruta dos adultos, de vinte anos ou mais, quando comparada a década de 1990-1999 com a de 1980-1989, no município de Rio de Janeiro (-87,5 por cem mil habitantes). E talvez não coincidentemente, a mortalidade por causas mal definidas foi a que mais cresceu no município do Rio de Janeiro, na comparação das mesmas décadas, nos adultos de vinte anos ou mais, em nível semelhante $(+84,6$ por cem mil habitantes) ao da queda por DAC. Portanto, é possível que um número ainda maior de óbitos mal definidos devesse ter sido incorporado àqueles por DAC e seus componentes, DIC e DCBV, como compensação, fazendo que as tendências de queda por esses grupos de causas não fossem tão pronunciadas no Rio de Janeiro, especialmente quando comparadas às experimentadas por São Paulo, Rio Grande do Sul e suas capitais. Esse ponto merece novas investigações que esclareçam as verdadeiras causas de óbitos mal definidos no passado e medidas que impeçam sua ocorrência no futuro.

Procura-se atribuir essa redução observada nos outros estudos ao controle dos fatores de risco cardiovascular conhecidos (hipertensão arterial, tabagismo, obesidade, diabete melito, dislipidemias, sedentarismo etc.). No Rio de Janeiro vimos que a prevalência da hipertensão arterial sistêmica, por exemplo, no estudo da Ilha do Governador, era de $38 \%$ da população adulta, e apenas $10 \%$ dos hipertensos tinham seu nível de PA controlado, enquanto o tabagismo variava de cerca de $30 \%$ na população de alta renda até $50 \%$ nos homens de baixo nível socioeconômico ${ }^{33}$. O Programa de Controle da Hipertensão Arterial do município do Rio de Janeiro ${ }^{34}$, que já alcançou quase trezentos mil pacientes registrados em mais de dez anos de atuação, controla apenas 19\% da população-alvo de hipertensos. 0 diabete, que ocorre em aproximadamente 8,7\% nas mulheres e 5,8\% nos homens acima de trinta anos de idade, também apresenta baixas taxas de controle, principalmente na população de nível socioeconômico mais baixo, em que cerca de dois terços desconhecem a sua condição ${ }^{35}$. A hipercolesterolemia atinge cerca de um terço da população adulta ${ }^{36}$. Sabe-se ainda que esses fatores de risco ocorrem mais freqüentemente na população de baixa renda e baixa escolaridade ${ }^{37}$.

Podemos ampliar a explicação da redução progressiva e discreta da mortalidade por DAC nas duas últimas décadas nos estados do RJ, SP e RS com as melhorias das condições de vida das populações, com mais municípios servidos por redes de água e esgoto e conseqüente redução das DIC.

Intenso processo educativo para a classe médica precisa ser desenvolvido para mostrar a importância do conhecimento correto da causa mortis para a tomada de decisões gerenciais em saúde. Ao mesmo tempo, os serviços de anatomia patológica, principalmente dos hospitais públicos, precisam ser reativados ou reaparelhados para poderem cumprir o seu papel fundamental na caracterização das causas de óbitos. Esse esclarecimento é fundamental, pois podemos supor também que ocorrem óbitos declarados como sendo por infarto do miocárdio ou por acidente vascular cerebral que se constituem em erros de declaração, por falta de comprovação.

\section{REFERÊNCIAS}

1. American Heart Association. Heart and Stroke Facts, 1998. Dallas: AHA, 1998.

2. Uemura K \& Pisa Z. Recent trends in cardiovascular disease mortality in 27 industrialized countries. WId HIth Statist Quart 1985; 38:142-62.

3. Mansur AP, Favarado D, Souza MFM, Avakian SD, Aldrighi JM, César
LAM, et al. Tendência da mortalidade por doenças circulatórias no Brasil de 1979 a 1996. Arq Bras Cardiol 2001; 76:497-503.

4. Ministério da Saúde. Guia de Vigilância Epidemiológica. $4^{\text {a }}$ ed. Brasília, 1998.

5. Ministério da Saúde. Guia de Vigilância Epidemiológica. $3^{a}$ ed. Brasília, 1996. 
6. Lotufo PA, Lolio CA. Tendência da mortalidade por doença isquêmica do coração no Estado de São Paulo: 1970 a 1989. Arq Bras Cardiol 1993; 61:149-53.

7. Lolio CA, Souza JMP, Laurenti R. Decline in cardiovascular disease mortality in the city of S.Paulo, Brazil, 1970 to 1983. Rev Saúde Pública 1986; 20:454-64.

8. Passos LC, Lopes AA, Lessa I, Sanches A, Santos-Jesus R. Tendência da mortalidade por infarto do miocárdio (1981 a 1996) na cidade de Salvador, Brasil. Arq Bras Cardiol 2000; 74:329-31.

9. Moraes SA, Rezende MHV, Freitas ICM. Tendência da mortalidade por doença isquêmica do coração no município de Goiânia - Brasil na série histórica entre 1980 e 1994. Arq Bras Cardiol 2000; 74:493-7.

10. Walter WJ. Changing U.S. lifestyle and declining vascular mortality. A retrospective. N Engl J Med 1983; 308:649-51.

11. Kuuslasmaa K, Tunstall-Pedoe H, Dobson A, Fortmann S, Sans S Tolonen $\mathrm{H}$, et al. Estimation of contribution of changes in classic risk factors to trens in coronary-event rates across the WHO MONICA Project populations. Lancet 2000; 355:675-87.

12. Collins R, Peto R, MacMahon S, Hebert P, Fiebach NH, Eberlein KA, et al. Blood pressure, stroke, and coronary heart disease Part 1. Prolonged differences in blood pressure: prospective observational studies corrected for the regression dilution bias. Lancet 1990; 335:827-38.

13. Eluf-Neto J, Lotufo PA, Lólio CA. Tratamento da hipertensão e declínio da mortalidade por acidentes vasculares cerebrais. Rev Saúde Pública de São Paulo 1990; 24:332-6.

14. Sytkowski PA, Kannel WB, D'Agostino RB. Changes in risk factors and the decline of mortality from cardiovascular disease. N Engl J Med 1990; 322:1635-41.

15. Secretaria de Saúde do Rio Grande do Sul [homepage na Internet] [acesso em dezembro de 2001]. Informações de Saúde. Morbidade e informações epidemiológicas. Disponível em <http:// www.saude.rs.gov.br>

16. Souza MFM, Timerman A, Serrano Jr CV, Santos RD, Mansur AP. Tendência do risco de morte por doenças circulatórias nas cinco regiões do Brasil no período de 1979 a 1996. Arq Bras Cardiol 2001; 77:562-68.

17. Moraes IHS, Santos SRFR. Informações em saúde: os desafios continuam. Ciências \& Saúde Coletiva 1998; III(1): 37-51

18. Instituto Brasileiro de Geografia e Estatística [homepage na Internet]. Censo 2000. [acesso em dezembro de 2001] Disponível em: http:/ /www.ibge.gov.br

19. Cláudio DM, Martins JM. Cálculo numérico populacional. São Paulo: Atlas, 1989.

20. Ministério da Saúde [homepage na Internet]. Secretaria Executiva. DATASUS. [acesso em dezembro de 2001]. Informações de Saúde. Morbidade e informações epidemiológicas. Disponível em: http:// www.datasus.gov.br

21. Organização Mundial de Saúde - Manual da Classificação Internacional de Doenças, Lesões e Causas de Óbitos, 9a Revisão,
1975, São Paulo, Centro da OMS para Classificação das Doenças em Português, 1978.

22. Organização Mundial de Saúde. Classificação estatística internacional de doenças e problemas relacionados à saúde: Classificação Internacional de Doenças -10 a Revisão. São Paulo: Edusp, 1995:1.

23. Statistics/Data Analysis -STATA Corporation: STATA, Version 7, University of Texas, USA, 2000, CD-ROM.

24. Thom TJ. International mortality from heart disease: rates and trends. Int J Epidemiol 1989; 18(supp.1):S20-S28.

25. Havlik RJ, Feinleib M, eds. Proceeding of the Conference on the Decline in Coronary Heart Disease Mortality. Bethesda, National Institute of Health 1979 (NIH publ 79-1610)

26. Chor D, Fonseca MJ, Andrade CR. Doenças Cardiovasculares. Comentários sobre a Mortalidade Precoce no Brasil. Arq Bras Cardiol 1995; 64:15-9.

27. Laurenti R, Gotlieb SL, Souza JMP, Fonseca LAM, Jorge MHPM. Características da mortalidade por doença isquêmica do coração em adultos de 15 a 74 anos no município de São Paulo. Arq Bras Cardiol $1981 ; 36: 85-9$.

28. Laurenti R. Epidemiologia das doenças cardiovasculares no Brasil. Arq Bras Cardiol 1982; 38:243-8.

29. Lolio CA, Laurenti R. Mortalidade por doença isquêmica do coração no município de São Paulo. Evolução de 1950 a 1981 e mudanças recentes na tendência. Arq Bras Cardiol 1986; 46:153-6.

30. Lolio CA, Lotufo PA, Lira AC, Zanetta DM, Massad E. Tendência da Mortalidade por Doença Isquêmica do Coração nas Capitais de Regiões Metropolitanas do Brasil, 1979-89. Arq Bras Cardiol 1995; 64:195-9.

31. Lolio CA, Laurenti R. Tendência da mortalidade por doença cerebrovascular em adultos maiores de 20 anos no município de São Paulo (Brasil), 1950 a 1981. Rev Saúde Pública S Paulo 1986; 20:243-6.

32. Portaria número 550 de 23 de Janeiro de 1990, publicada no Diário Oficial de 29 de janeiro de 1990.

33. Klein $\mathrm{CH}$, Silva NAS, Nogueira AR, Campos LHS, Bloch KV.Hipertensão arterial na Ilha do Governador-Rio de Janeiro-Brasil II. Cad. Saúde Pública (reports in Public Health) 1995; 11:389-94.

34. Ramos ACMF, Seixas TC, Rocha CRM, Monteiro GT, Farias AMR. Avaliação Transversal do Controle da Hipertensão Arterial Sistêmica em Programa de Larga Escala. JBM 2001; 81:63-70.

35. Franco L. Diabetes in Brazil: a review of recent survey data. Ethnicity and Disease 1992; 2:158-65.

36. Souza LJ, Chalita FEB, Reis AFF, Teixeira CL, Giocavate Neto C, Bastos DA, et al. Obesidade e fatores de risco cardiovascular em Campos-RJ. Arq Bras Cardiol 2002; 79(supl III):70.

37. Brandão AA, Magalhães EC, Pozzan R, et al. O nível socioeconômico e os fatores de risco cardiovasculares em jovens acompanhados por 10 anos. Estudo do Rio de Janeiro. Arq Bras Cardiol 2002; 79(supl III): 18. 